\title{
Isolation and Characterization of Cyanobacterial Extracellular Vesicles
}

\author{
Steven J. Biller ${ }^{*}$, , María del Carmen Muñoz-Marín ${ }^{*}, 2$, Steeve Lima ${ }^{3,4,5}$, Jorge Matinha-Cardoso ${ }^{3,4}$, Paula Tamagnini ${ }^{3,4,6}$, Paulo \\ Oliveira $^{3,4,6}$ \\ ${ }^{1}$ Department of Biological Sciences, Wellesley College ${ }^{2}$ Departamento de Bioqúimica y Biologíia Molecular, Campus de Excelencia Internacional, \\ Agroalimentario, Universidad de Ćordoba ${ }^{3}$ i3S - Instituto de Investigação e Inovação em Saúde, Universidade do Porto ${ }^{4}$ IBMC - Instituto de Biologia \\ Molecular e Celular, Universidade do Porto ${ }^{5}$ MCbiology Doctoral Program, ICBAS - Instituto de Ciências Biomédicas Abel Salazar, Universidade do \\ Porto ${ }^{6}$ Departamento de Biologia, Faculdade de Ciências, Universidade do Porto \\ *These authors contributed equally
}

\section{Corresponding Authors}

Steven J. Biller

sbiller@wellesley.edu

María del Carmen Muñoz-Marín

b32mumam@uco.es

Paulo Oliveira

paulo.oliveira@ibmc.up.pt

\section{Citation}

Biller, S.J., Muñoz-Marín, M.d.C., Lima, S., Matinha-Cardoso, J., Tamagnini, P., Oliveira, P. Isolation and Characterization of Cyanobacterial Extracellular Vesicles. J. Vis. Exp. (180), e63481, doi:10.3791/63481 (2022).

\section{Date Published}

February 3, 2022

DOI

$10.3791 / 63481$

URL

jove.com/video/63481

\section{Abstract}

Cyanobacteria are a diverse group of photosynthetic, Gram-negative bacteria that play critical roles in global ecosystems and serve as essential biotechnology models. Recent work has demonstrated that both marine and freshwater cyanobacteria produce extracellular vesicles - small membrane-bound structures released from the outer surface of the microbes. While vesicles likely contribute to diverse biological processes, their specific functional roles in cyanobacterial biology remain largely unknown. To encourage and advance research in this area, a detailed protocol is presented for isolating, concentrating, and purifying cyanobacterial extracellular vesicles. The current work discusses methodologies that have successfully isolated vesicles from large cultures of Prochlorococcus, Synechococcus, and Synechocystis. Methods for quantifying and characterizing vesicle samples from these strains are presented. Approaches for isolating vesicles from aquatic field samples are also described. Finally, typical challenges encountered with cyanobacterial vesicle purification, methodological considerations for different downstream applications, and the trade-offs between approaches are also discussed.

\section{Introduction}

Extracellular vesicles (EVs) are spherical structures, ranging between $\sim 20-400 \mathrm{~nm}$ in diameter, released by virtually all organisms into their surrounding environment ${ }^{1,2,3}$. Vesicles are delimited by a lipid bilayer and cannot replicate themselves. In Gram-negative bacteria, these structures are thought to primarily arise by 'blebbing' of small portions from 
the outer membrane. Still, other processes, including flagellar motion, cell lysis, and secretion of both inner and outer membrane material, can produce vesicles as well ${ }^{4,5}$. EVs can contain various biomolecules, including lipids, soluble and membrane proteins, nucleic acids, and metabolites, and can transport this material between cells $s^{4,5,6}$. Given these features, EVs are being studied to understand their possible roles in a wide range of biological processes, including cellular communication, biofilm formation, horizontal gene transfer, host-phage dynamics, and nutrient exchange ${ }^{4,6}$.

Cyanobacteria are a large and diverse group of Gramnegative bacteria, including unicellular and filamentous organisms. They are of interest from many perspectives, including understanding their physiology and diversity ${ }^{7,8}$, the critical ecosystem functions they serve ${ }^{9,10}$, and their utility for biotechnology 11,12 . Cyanobacteria are found in a wide variety of habitats, either as free-living organisms in marine, freshwater, and terrestrial environments, or in symbiotic associations with mosses, ferns, plants, or in lichens and sponges ${ }^{13}$. They serve as crucial primary producers in aquatic ecosystems, producing oxygen and organic carbon through oxygenic photosynthesis ${ }^{9,10}$, and some are capable of fixing atmospheric nitrogen as well ${ }^{7}$. Marine and freshwater cyanobacteria, including Prochlorococcus, Synechococcus, and Synechocystis, produce EVs under laboratory conditions ${ }^{14,15,16}$, and cyanobacterial vesicles can also be found in natural environments ${ }^{14,17}$. The biological and ecological functions of cyanobacterial vesicles are unknown, but further research in this area is likely to provide new insights into questions on cyanobacterial physiology, differentiation, communication strategies, evolution, and trophic interactions. In addition, the ability of cyanobacterial EVs to carry diverse categories of biomolecules may have commercial applications ${ }^{18,19}$.

The present protocol describes methods for isolating and characterizing vesicles from cyanobacterial cultures and field samples to enable and encourage a broader examination of cyanobacterial extracellular vesicle biology. While the workflow described here is analogous to protocols for isolating and characterizing EVs from other bacteria, cyanobacterial cultures and field samples typically contain lower cell and vesicle concentrations than are commonly observed with host-associated or pathogenic model systems $20,21,22$. Thus, studies of cyanobacterial EVs require special considerations and optimizations for cultivation and vesicle isolation, which vary further between strains and media backgrounds. As no single protocol will work equally well for all strains, growth conditions, and downstream applications, we provide multiple options and discuss the trade-offs involved so that researchers may determine the approaches most appropriate for addressing their experimental questions.

\section{Protocol}

The cyanobacterial strains are obtained from several culture collections (see Discussion for details).

\section{Cyanobacterial cultivation}

1. Cultivate marine cyanobacteria Prochlorococcus and Synechococcus following the steps below.

1. Cultivate Prochlorococcus and Synechococcus strains in polycarbonate flasks using an appropriate medium such as Pro99 or SN (see Table of Materials). For details, please see previously published References ${ }^{23,24}$. 
2. Acclimate the cultures by growing them across two to three transfers (1:20 dilutions of culture into fresh media for each passage) under the desired temperature and irradiance conditions required for the experiment.

NOTE: Typical growth conditions ${ }^{23,25}$ include temperatures between $15-30{ }^{\circ} \mathrm{C}$ at irradiance between 8-150 $\mu$ mol photons $\mathrm{m}^{-2} \mathrm{~s}^{-1}$, but individual strain tolerances and optima vary widely ${ }^{26}$ and need to be set based on guidance from the relevant culture collection instructions or literature. Monitor growth rate using culture fluorescence or flow cytometry counts ${ }^{23}$, and ensure that cultures grow at a consistent steady-state exponential rate before beginning the experiment.

3. Carry out a set of successive gradual transfers from smaller to larger volumes once cells reach late exponential phase (e.g., $20 \mathrm{~mL}<250 \mathrm{~mL}<2 \mathrm{~L}<10$ $L<20 \mathrm{~L})$.

NOTE: Large volume cultures cannot be directly inoculated from small amounts of starting culture. Note that larger cultures may require the addition of buffers (such as $1 \mathrm{mM}$ HEPES, pH 7.5 or 3.75 mM TAPS, pH 8), along with supplementary sodium bicarbonate or bubbling with sterile $\mathrm{air}^{24}$.

2. Cultivate freshwater cyanobacterium Synechocystis sp. PCC 6803.

1. Prepare a culture of Synechocystis sp. PCC 6803 to be used as inoculum by cultivating it with aeration (bubbling air at $1 \mathrm{~L} / \mathrm{min}$ ), at $30^{\circ} \mathrm{C}$, under a $16 \mathrm{~h}$ light (photons count of $50 \mu \mathrm{mol} \mathrm{m}{ }^{-2} \mathrm{~s}^{-1}$ ) / $8 \mathrm{~h}$ dark regime, up to an optical density at $730 \mathrm{~nm}$ (OD730) of 1.0 (exponential phase) in BG11 medium ${ }^{27}$.
2. Inoculate $30 \mathrm{~mL}$ of this culture Synechocystis $\mathrm{sp}$. PCC 6803 in $570 \mathrm{~mL}$ of BG11 medium to an OD730 of 0.05 in $1 \mathrm{~L}$ glass gas washing bottles.

3. Allow cells to grow with aeration, at $30^{\circ} \mathrm{C}$, under a $16 \mathrm{~h}$ light (photon count of $50 \mu \mathrm{mol} \mathrm{m} \mathrm{m}^{-2} \mathrm{~s}^{-1}$ ) / $8 \mathrm{~h}$ dark regime, up to a final OD730 of 1.0-1.5.

\section{Separation of cyanobacterial biomass from the small particle (vesicle) fraction}

NOTE: First, separating cells from the supernatant through centrifugal pelleting of cells is recommended. However, when culture volumes are too large for this to be feasible, it is possible to skip centrifugation and proceed directly to filtering whole cultures using capsule filtration (step 2.4).

1. Perform centrifugation to separate cells (best for volumes up to $\sim 4 \mathrm{~L}$, depending on available centrifuge capacity).

1. Autoclave or thoroughly wash and clean the centrifuge bottles using Type I ultrapure grade water (see Table of Materials), ensuring that no residual soap or other material remains.

2. Load culture samples into an appropriate number of centrifuge bottles and balance them.

3. Spin cultures for $>10,000 \times g$ at $4{ }^{\circ} \mathrm{C}$ for $10 \mathrm{~min}$. If the supernatant remains visibly turbid, increase centrifugation conditions to $20 \mathrm{~min}$ at maximum speed and retry.

4. Carefully decant or pipet supernatant into a clean vessel and proceed to one of the following filtration options mentioned in steps 2.2-2.4.

2. Option 1: perform syringe filtration using $0.2 \mu \mathrm{m}$ filters (for sample volumes up to $\sim 50 \mathrm{~mL}$ ). 
1. Fill a sterile $50 \mathrm{~mL}$ syringe with a largely cellfree culture medium, and filter it through a 0.2 (or 0.45) $\mu \mathrm{m}$ polyethersulfone (PES) filter (see Table of Materials). Collect the filtrate in a clean, sterilized receptacle.

2. Repeat this step until the filter is clogged (e.g., it becomes difficult to push with the syringe). Replace with a new filter, and proceed until the sample is thoroughly filtered.

3. Option 2: perform $0.2 \mu \mathrm{m}$ vacuum filtration (up to $\sim 4 \mathrm{~L}$ ).

1. Thoroughly wash and clean the vacuum apparatus using Type I - ultrapure grade water, connecting it to a vacuum trap and pump (see Table of Materials).

2. Insert a $0.2 \mu \mathrm{m}$ filter of an appropriate diameter and clamp the vacuum apparatus.

3. Add a small amount of culture sample, ensuring that the vacuum pressure remains $<10 \mathrm{psi}$.

4. Continue filtering the culture in small increments until complete. If the filtration rate slows significantly, stop the vacuum and replace the filter.

5. Collect the $<0.2 \mu \mathrm{m}$ fraction containing vesicles into a clean container.

4. Option 3: perform $0.2 \mu \mathrm{m}$ capsule filtration (for sample volumes $>\sim 4 \mathrm{~L}$ )

1. Clean appropriately sized flexible tubing and collection vessel by washing with water and mild detergent. Rinse the material with distilled and deionized water.

NOTE: Prior to utilization, the material should be rinsed with Type I - ultrapure grade water.

2. Place the culture to be filtered in a secure, elevated location with the final collection vessel below.
Connect a $0.2 \mu \mathrm{m}$ capsule filter (see Table of Materials) outflow port to one piece of tubing and place it in a collection vessel.

3. Place another tubing into the sample, begin a gravity siphon by pulling some sample into the tubing with a syringe, and connect the tubing to the capsule filter. Use the vent to release excess air and fill the chamber with supernatant.

4. Allow material to move through the capsule until the sample is thoroughly filtered. If the flow rate becomes too slow $\left(<\sim 1 / 10^{\text {th }}\right.$ the starting flow rate, or coming out dropwise as opposed to a continuously flowing stream), wait or apply gentle force with a peristaltic pump until backpressure increases. Alternatively, partially restore the flow rates by temporarily disconnecting the filter, backflushing accumulated biomass from the inflow side with ultraclean water until the material is no longer visibly turbid, and then restart the filtration process.

\section{Concentrating the vesicle sample}

1. Option 1: perform centrifugal ultrafiltration for concentrating small $(<500 \mathrm{~mL})$ volume of samples.

1. Rinse the $15-20 \mathrm{~mL}$ ultrafiltration centrifugal concentrators of choice with Type I - ultrapure grade water.

2. Load the concentrators into the centrifuge and spin at $4,400 \times g$ at $4{ }^{\circ} \mathrm{C}$. Discard the run through and repeat this step at least twice more.

3. Load the supernatant sample into water-rinsed concentrators and spin under the same conditions. Depending on experimental goals, the sample filtrate can be discarded or collected for further 
concentration (with concentrators with a nominal molecular weight limit of $3 \mathrm{kDa}$ ) and analysis.

4. Repeat this step until the sample is concentrated to a final volume of $\sim 15-30 \mathrm{~mL}$.

2. Option 2: perform tangential flow filtration (TFF) for concentrating a large $(>500 \mathrm{~mL})$ volume of sample.

1. Set up the TFF apparatus according to the manufacturer's guidelines (see Table of Materials). Attach a peristaltic pump to the intake line and place adjustable clamps on the retentate line. Sanitize the TFF as recommended by the manufacturer, and then flush the device with $1 \mathrm{~L}$ of Type I - ultrapure grade water.

NOTE: The intake and retentate lines should be placed into a clean sample reservoir (glass bottle). Filtrate line(s) should be directed into a waste vessel or sink.

2. Add $<0.2 \mu \mathrm{m}$ filtrate into the sample reservoir. Slowly increase pump speed and backpressure levels on the retentate line to increase output from the filtrate lines.

3. Continue to run the TFF, refilling the reservoir with culture supernatant as the material is removed. Avoid allowing the intake line to come out of the sample during processing to avoid introducing air bubbles. Ensure that feed pressure does not exceed $\sim 10$ psi and that the retentate flows out of the TFF at a consistent pace back into the reservoir.

4. Stop concentrating the sample once the volume in the reservoir reaches the lowest possible amount needed to maintain flow into the intake line without introducing air bubbles.
5. Close the outflow line with a clamp. Remove the backpressure on the retentate line and recirculate the concentrated supernatant through the filter for $\sim 10 \mathrm{~min}$, reducing the recirculation rate to $20-40 \mathrm{~mL} /$ min to maximize recovery.

6. Move the retentate line into a clean vessel, remove the intake line from the sample, and collect the concentrated material. Recover any remaining material in the sample reservoir using a pipette.

7. Filter the concentrated supernatant through a $0.2 \mu \mathrm{m}$ syringe filter (step 2.2) to ensure no cells remain. NOTE: Step 3.2.7 is optional.

8. If needed, store the final concentrate at $4{ }^{\circ} \mathrm{C}$ for $\sim 3$ weeks before moving to vesicle purification (step 4).

\section{Vesicle isolation and purification}

1. Pellet directly by ultracentrifugation following the steps below.

1. Place the concentrated $<0.2 \mu \mathrm{m}$ culture sample in a clean ultracentrifuge tube. Add clean media or buffer as necessary to ensure that the tube is completely filled.

NOTE: If the final culture sample is too large to fit in one tube, either pellet the material in multiple tubes to be combined before the wash steps or pellet serially in the same tube.

2. If needed, create a balance and spin at $\sim 100,000 x$ $g$ for $3 \mathrm{~h}$ at $4^{\circ} \mathrm{C}$.

3. Carefully remove the supernatant with a pipette. While cyanobacterial vesicle pellets can show some coloration, they are frequently invisible. 
4. Wash the pelleted material by adding fresh culture media or wash buffer such as $1 x$ phosphatebuffered saline (PBS) (see Discussion) to the ultracentrifuge tube, mix by gentle pipetting, and spin again as in step 4.1.2. Repeat the wash process a second time.

5. Resuspend the final pellet in fresh culture by repeatedly but gently pipetting up and down around the bottom of the tube with a $1 \mathrm{~mL}$ pipette. Transfer to a clean vessel.

2. Perform density gradient ultracentrifugation.

1. Prepare iodixanol stocks (see Table of Materials) by making a $4 x$ concentrated version of the buffer background desired for the sample (see Discussion).

2. Mix one part of the $4 x$ buffer with three parts of $60 \%$ iodixanol stock to make a $45 \%$ iodixanol solution.

3. Dilute $45 \%$ iodixanol with volumes of $1 \mathrm{x}$ buffer to create stocks of gradient media at $40 \%, 35 \%$, $30 \%, 25 \%, 20 \%, 15 \%$, and $10 \%$ final iodixanol concentrations.

NOTE: The total amount needed will vary with the capacity of the ultracentrifuge rotor/tubes.

4. Set up an ultracentrifuge density gradient by carefully overlaying equal amounts of $45 \%, 40 \%$, $35 \%, 30 \%, 25 \%, 20 \%, 15 \%, 10 \%$, and $0 \%$ ioxidinol into an ultracentrifuge tube such that the entire volume of the tube is utilized.

NOTE: The extracellular vesicle sample to be purified should be placed at the top of the gradient as part of the $0 \%$ iodixanol layer. If the final volume of the vesicle sample exceeds the size of the $0 \%$ layer, mix any excess vesicle sample with enough
$45 \%$ iodixanol and/or buffer to generate the required volume of the $10 \%$ or higher concentration optiprep stocks, and use these in the corresponding location in the gradient.

5. Spin the gradient at $\sim 100,000 \times g$ at $4{ }^{\circ} \mathrm{C}$ for $6 \mathrm{~h}$.

6. Collect fractions (typically $0.5 \mathrm{~mL}$ each for a $\sim 4.5$ $\mathrm{mL}$ gradient) by careful pipetting or using a fraction collector (see Table of Materials).

7. Determine the density of each fraction (in $\mathrm{g} / \mathrm{mL}$ ) using an analytical balance and calibrated pipette to measure the weight of a known volume of sample. Remove the sample from the tube, determine the weight, and return the sample directly.

8. Dilute individual fractions in a new ultracentrifuge tube with clean buffer and wash the material as mentioned in steps 4.1.2-4.1.4. Alternatively, use dialysis or ultrafiltration columns to recover particles. NOTE: Cyanobacterial extracellular vesicles typically migrate to buoyant densities of $\sim 1.14-1.19$ $\mathrm{g} / \mathrm{mL}$ in iodixanol.

3. Store the vesicles at $4{ }^{\circ} \mathrm{C}$ if they are to be used within $1-3$ weeks. Freeze the vesicles at $-20^{\circ} \mathrm{C}$ or $-80^{\circ} \mathrm{C}$ if they are not used for longer than that period.

\section{Characterization of the isolated vesicles}

1. Perform negative stain transmission electron microscopy (see Table of Materials) (TEM; particle size, structure, purity).

1. To improve the image quality, glow discharge the surface of a former-coated TEM grid using a glow discharge system according to the manufacturer's guidelines (see Table of Materials). 
2. Carefully apply $\sim 5 \mu \mathrm{L}$ of the vesicle sample and let sit for $5 \mathrm{~min}$.

NOTE: Samples with vesicle concentrations $>10^{9}$ $\mathrm{mL}^{-1}$ will typically give the best results; more dilute samples will have few vesicles per image.

3. Remove the sample by touching the edge of a grid to a piece of clean filter paper.

4. Pipet a $20-50 \mu \mathrm{L}$ drop of $2 \%$ uranyl acetate (see Table of Materials) onto a flat surface covered by plastic film, and place the grid floating on top of it for $2 \min$

5. Remove uranyl acetate using filter paper and float briefly on a drop of ultrapure water to wash. Repeat the water wash a second time.

NOTE: The final dry grid is ready for visualization after step 5.1.5.

2. Perform ultra-thin section staining for TEM (particle size, internal structure).

1. Resuspend the pellet from step 4.1 .5 with $1 \mathrm{~mL}$ of $2.5 \%$ glutaraldehyde in $0.1 \mathrm{M}$ sodium cacodylate buffer $(\mathrm{pH}$ 7) (see Table of Materials) and fix the sample for $2 \mathrm{~h}$ at $4{ }^{\circ} \mathrm{C}$.

2. Spin at $\sim 100,000 \times g$ for $1 \mathrm{~h}$ at $4{ }^{\circ} \mathrm{C}$.

3. Wash the pellet carefully with $0.1 \mathrm{M}$ sodium cacodylate buffer ( $\mathrm{pH} 7)$, and then spin at $\sim 100,000$ $\mathrm{xg}$ for $1 \mathrm{~h}$ at $4{ }^{\circ} \mathrm{C}$.

4. Fix the pellet with $1 \mathrm{~mL}$ of $1 \%$ osmium tetroxide (see Table of Materials) (prepared in $0.1 \mathrm{M}$ sodium cacodylate buffer) for $1 \mathrm{~h}$, and then wash as in step 5.2.3.
5. Dehydrate the sample with $1 \mathrm{~mL}$ of an ascendant series of ethanol $(50 \%, 70 \%, 90 \%$, and two times in absolute ethanol) in steps of 10 min each at $4{ }^{\circ} \mathrm{C}$.

6. Add $250 \mu \mathrm{L}$ of epoxy resin (see Table of Materials) mixed with absolute ethanol (1:1) to the pellet and incubate overnight at $4{ }^{\circ} \mathrm{C}$.

7. Transfer the pellet to $500 \mu \mathrm{L}$ of pure resin for $24 \mathrm{~h}$. Then, incubate the resin at $65{ }^{\circ} \mathrm{C}$ for $48 \mathrm{~h}$.

NOTE: The resin is now ready for sectioning by ultramicrotome, following the manufacturer's instructions (see Table of Materials).

8. Stain the grids containing sections with $2 \%$ uranyl acetate (in $50 \%$ ethanol) for $5 \mathrm{~min}$. Wash the slides gently under running deionized water for 10-15 s each. Place a drop of lead citrate (see Table of Materials) and stain for another 5 min.

9. Wash as before, remove the water by blotting the grid on a filter paper, and air-dry the grids. Visualize by TEM according to the manufacturer's guidelines (see Table of Materials).

3. Perform nanoparticle tracking analysis (NTA) (particle size, concentration).

1. Using lens paper, carefully wipe any dust or visible material off the optical flat. Check whether all O-rings and other seals are clean and intact. Turn on the instrument and start up the software.

2. Using a clean syringe, fill the chamber with ultra-pure water. Ensure that no air bubbles are present.

3. Click on Start Camera and visualize the optimal region of the chamber about the 'thumbprint'. Adjust the microscope stage horizontally or vertically as needed so that signal intensity is even across the 
imaged region. Move the imaging region horizontally relative to the thumbprint (to the right, toward the vertical line), finding a region nearby with low background signal.

4. Slide the Screen Gain and Camera Level sliders to maximum, and then decrease to the lowest level to see the dimmest particles.

NOTE: Typical settings for cyanobacterial vesicles utilize a Screen Gain of 7 and Camera Level between 10-12.

5. Adjust the focus as necessary to ensure that particles are roughly equally visible across the field of view. Continue to push ultra-clean water through the chamber until you have visually confirmed that the chamber is clean.

6. Remove residual water from the chamber using a different syringe.

7. Examine a sample of the media/buffer your sample is in to determine the background particle concentration by filling the chamber with media/ buffer using a clean $1 \mathrm{~mL}$ syringe.

8. Select Standard measurement from the SOP dropdown box. Change settings to collect at least three technical replicates of $60 \mathrm{~s}$ videos each. Press Create and Run Script and follow the prompts. Push $\sim 100 \mu \mathrm{L}$ of sample into the chamber between replicates.

9. When the acquisition is complete, remove the residual buffer with a syringe, flush $3-5 \mathrm{~mL}$ of ultrapure water through the chamber, and remove the remaining water.

10. Add the vesicle sample to the chamber using a 1 $\mathrm{mL}$ syringe and confirm the acquisition settings. If particle count is not within the instrument's linear range (typically between $20-80$ particles/frame), the sample will need to be diluted or concentrated. Push at least $500 \mu \mathrm{L}$ of sample into the chamber before collecting data.

11. Collect video data as in step 5.3.8, making sure to clean the chamber with ultra-pure water between different samples.

12. Collect all subsequent samples from the same organism/experiment using identical camera settings to ensure comparability of data; changes in the Camera Level setting can have a notable effect on the final values obtained.

13. Determine particle parameters using the analysis section of the software. Select Analysis > Open Experiment, and load the sample file. Select Process Selected Files and wait for the analysis to complete. Ensure to use the same Detection Threshold for all subsequent samples from the same experiment.

NOTE: As cyanobacterial vesicles are frequently dim, the Detection Threshold will typically need to be adjusted to the most sensitive (lowest) value.

4. Perform dynamic light scattering (DLS) (particle size, zeta potential).

1. Filter through a $0.2 \mu \mathrm{m}$ pore size filter for any sample that goes into the DLS to screen out large particles that interfere with measurements.

2. Add $1 \mathrm{~mL}$ of the media/buffer into a clean cuvette to examine possible aggregations or other nanoparticles coming from the media. Put the cuvette with the frosted side on the left into the micro 
sampler, and close the lid. Let the sample equilibrate for at least $5 \mathrm{~min}$.

3. Enter the refractive index of the material and the material absorption if they differ significantly from the defaults for water.

NOTE: The material properties would have very little influence if the vesicles are smaller than 100 $\mathrm{nm}$. When measuring the surface potential (zeta potential) of EVs, vesicles should be resuspended in the original growth media.

4. Click on Instrument Control Panel to check the readings and start data acquisition by clicking on the green icon. If the values look good and your media/buffer does not contain aggregations or other particles, you are now ready to measure your sample.

5. Add $1 \mathrm{~mL}$ of vesicle sample into a clean cuvette and collect data as mentioned in step 5.4.4. Collect at least three technical replicates for 10 min each.

5. Perform lipopolysaccharide (LPS) analysis to confirm that the Gram-negative EVs are present in the sample.

1. Heat denature vesicle sample at $95{ }^{\circ} \mathrm{C}$ for $10 \mathrm{~min}$ in standard 1x Laemmli sample buffer (see Table of Materials).

2. Incubate with $0.2 \mathrm{U}$ of type XIV protease from Streptomyces griseus (see Table of Materials) at $37{ }^{\circ} \mathrm{C}$ for $30 \mathrm{~min}$ to remove contaminating glycoproteins.

3. Separate treated samples by electrophoresis on denaturing $16 \%(\mathrm{w} / \mathrm{v})$ SDS-polyacrylamide gels following the previously published protocol ${ }^{16}$.
4. To detect LPS, stain the gel either with commercially available staining kits or with a modified silver staining technique ${ }^{28}$. Quantify the relative LPS abundance by densitometry analysis of stained gels as per previously published References ${ }^{29,30}$.

\section{Vesicle production rate measurements}

1. Using nanoparticle tracking analysis as in step 5.3, or equivalent technology, measure the particle concentration in the growth media for the experiment. If a high particle count is found, filter through a $0.1 \mu \mathrm{m}$ pore filter and recheck.

NOTE: To minimize the error, the media background particle concentration should be less than $10 \%$ of the particle concentration found in cultures at the lowest density.

2. Acclimate the culture by growing cells for at least two successive serial transfers under the media and environmental conditions desired for the experiments as in step 1.1.2. Cultures need to be transferred while still in the exponential growth phase. Ensure that the measured culture growth rates are consistent across transfers; if not, continue transferring cultures until growth rates are reproducible.

3. Collect time-course samples upon inoculation and regularly timed intervals across the growth curve into the early stationary phase. Stagger time points to have a minimum of 3-4 samples collected across the full range of exponential growth. To sample at each time point, perform the following steps.

1. Filter $1 \mathrm{~mL}$ or more of culture directly through a clean, sterile $0.2 \mu \mathrm{m}$ syringe filter and save the filtrate to measure vesicles concentration as in step 
5.3. Freeze the time-course samples if desired. Use relative fluorescence to follow culture growth dynamics.

2. Save a sample of the whole culture to determine population size using a method appropriate for the organism (flow cytometry; colony plating; or otherwise).

4. Obtain measurements of cells and vesicle-like particles (per $\mathrm{mL}$ ) at each timepoint.

5. Identify the timepoints which occurred during the exponential growth phase. To calculate $r$, the number of vesicles produced per cell per generation, between two points during exponential growth (typically the beginning and end of the time-course), use the equation provided in Supplementary File 1.

NOTE: This method is only valid during steadystate growth conditions; underlying assumptions and derivation of the calculation are detailed in Reference ${ }^{14}$.

\section{Representative Results}

Figure 1 presents an overview of the cyanobacterial vesicle isolation process, highlighting key aspects of the protocol described here. Of particular note are the steps detailing separation of cyanobacterial biomass from the small particle (vesicle) fraction, concentrating the vesicle sample and vesicle isolation and purification (Figure 1, steps 2 to 4), which are critical to obtaining reproducible preparations of vesicles. Figure 2 presents representative results from the isolation and characterization of extracellular vesicles from the cyanobacterium Synechocystis sp. PCC 6803. The outer membrane of this cyanobacterium has been shown to contain carotenoids ${ }^{31}$, which confer a characteristic orange coloration to vesicles' samples collected by direct pelleting through ultracentrifugation (Figure 2A). Once the vesicle pellet is resuspended, vesicles can be examined by transmission electron microscopy (TEM), either by negatively staining samples with uranyl acetate (Figure 2B) or by observation of ultrathin sections (Figure 2C). Vesicle size distribution and concentration can be assessed using dynamic light scattering (DLS) (Figure 2D) and nanoparticle tracking analyses (NTA) (Figure 2E). With the described protocol, typically $\sim 3.5 \pm 1.0 \times 10^{8}$ nanoparticles per $\mathrm{mL}$ were obtained in an exponentially growing culture of Synechocystis sp. PCC 6803 at an OD730 of 1.0. Biochemical analyses of isolated EVs can be carried out to complement the physical characterization of the isolated vesicles. As an example, purified Synechocystis sp. PCC 6803 vesicles were separated on an SDS-polyacrylamide gel and stained for lipopolysaccharides (LPS; Figure 2F). As LPS are specific to the outer membrane ${ }^{32}$, LPS detection can validate the presence of membrane-bound vesicles and serve as a marker for analyzing relative vesicle content between preparations from the same strain ${ }^{33}$. Inspection of the relative abundance of low vs. high molecular weight LPS can indicate changes in vesicle composition between samples ${ }^{34,35}$. 


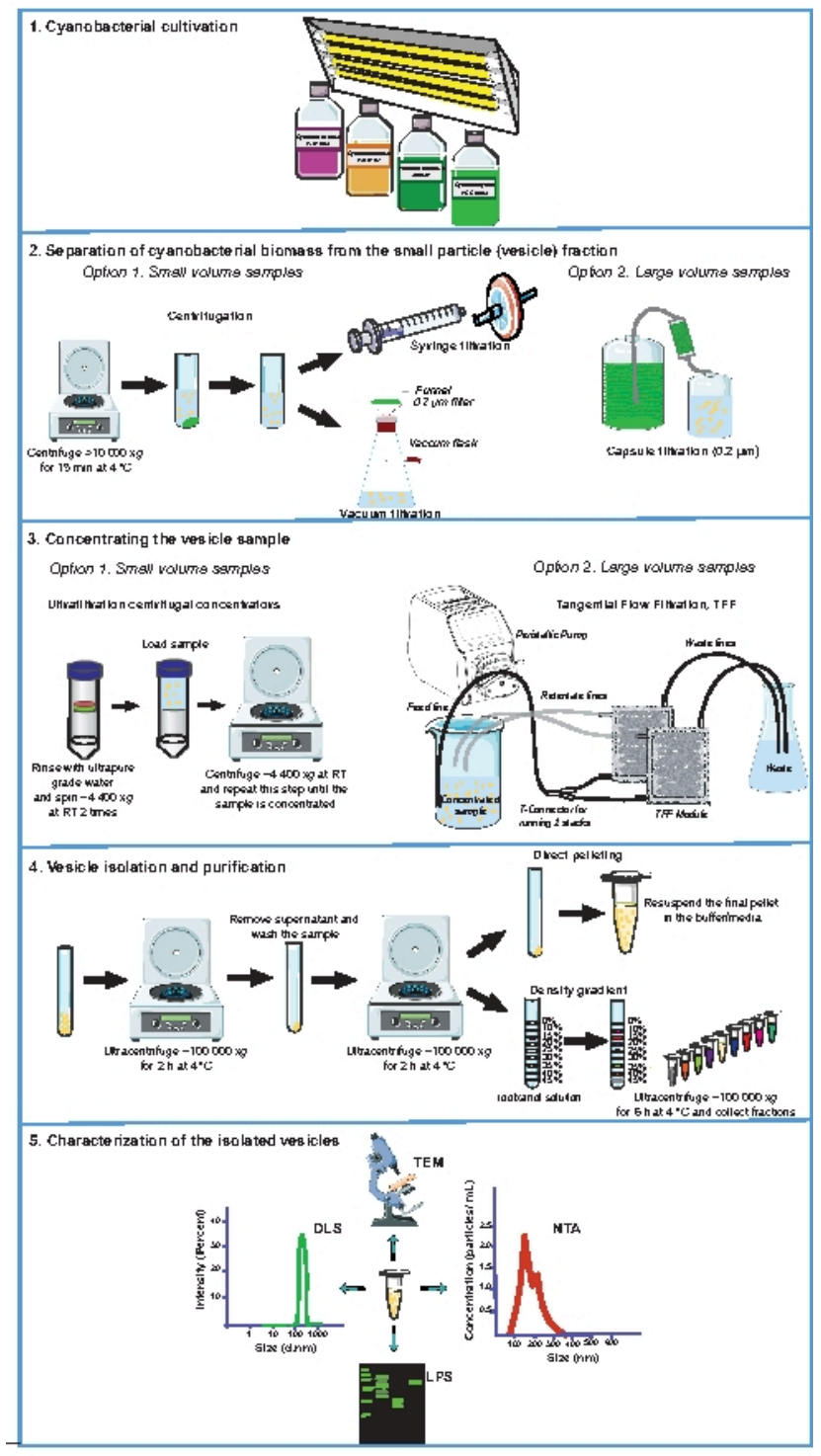

Figure 1: Experimental procedures used for cyanobacterial EV isolation and characterization. Schematic representations of growing cultures (1) and separation of cyanobacterial biomass from the growth medium (2). Cellfree samples containing vesicles are concentrated (3), and then the EVs are isolated and purified (4). Depending on the application, vesicle preparations can be characterized using one or a combination of transmission electron microscopy (TEM), nanoparticle tracking analysis (NTA), dynamic light scattering (DLS), and lipopolysaccharide (LPS) profiling (5). RT, room temperature. Please click here to view a larger version of this figure. 


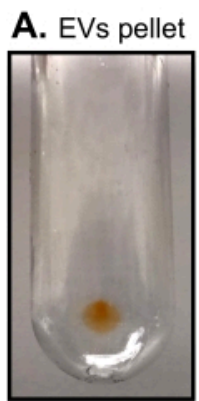

B. TEM - Negative staining

C. TEM - Ultrathin sections
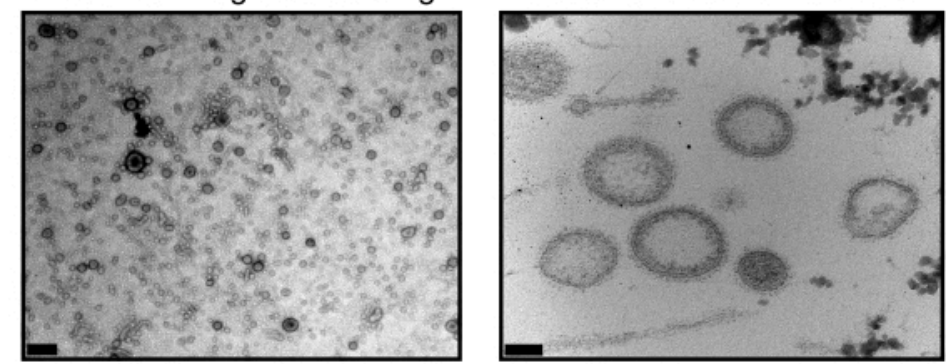

D. DLS

E. NTA

F. LPS
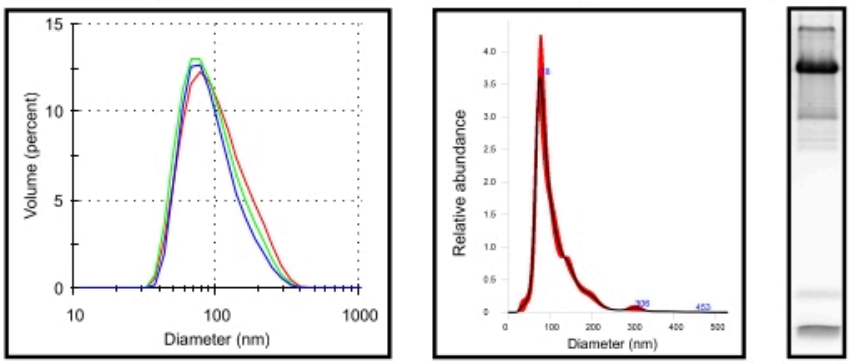

Figure 2: Representative results of the extracellular vesicle isolation and characterization for the cyanobacterium Synechocystis sp. PCC 6803. (A) Photograph of the extracellular vesicle pellet (EVs pellet) obtained after ultracentrifugation of the concentrated cell-free extracellular medium from a $600 \mathrm{~mL}$ Synechocystis sp. PCC 6803 culture grown to an OD730 of 1.0-1.5. Note the typical orange coloration of the vesicle pellet derived from the carotenoids found in the outer membrane. (B,C) Transmission electron microscopy (TEM) photographs of negatively-stained (B) and ultrathin sections (C) of Synechocystis sp. PCC 6803 vesicle samples. Scale bars: $200 \mathrm{~nm}$ and $50 \mathrm{~nm}$, respectively. Samples were visualized on a transmission electron microscope operated at $80 \mathrm{kV}$. (D) Typical dynamic light scattering (DLS) plot depicting the distribution of vesicle volume as a function of vesicle diameter (in $\mathrm{nm}$ ). Colored lines indicate data from three technical replicate measurements of the same sample. (E) Representative nanoparticle tracking analysis (NTA) data of Synechocystis extracellular vesicle size distribution (in $\mathrm{nm}$ ). The black line indicates the mean of three technical replicates, and the red lines represent the standard error of the mean. (F) Lipopolysaccharides (LPS) profile was detected after separating the vesicle preparation by electrophoresis on a 16\% (w/v) SDS-polyacrylamide gel and staining with a commercial LPS staining kit. Lowand high-molecular-weight LPS, corresponding to rough- and smooth-LPS forms, respectively, are detectable. Please click here to view a larger version of this figure.

Supplementary File 1: Equation to calculate $r$. The number of vesicles produced per cell per generation between two points during exponential growth (typically the beginning and end of the time course) is determined using this equation.

Please click here to download this File.

Discussion

General considerations 
The protocol (Figure 1) is presented as a set of options to highlight that there is no 'one-size-fits-all' method for working with cyanobacterial extracellular vesicles. Interested researchers can utilize the sections of this protocol that are compatible and appropriate for their particular model organism, experimental questions/goals, and equipment availability. All vesicle isolation approaches involve tradeoffs and will unavoidably result in some degree of bias. While one should seek to minimize this whenever possible, the most crucial consideration is to ensure that the detailed methodology used is reported according to appropriate MISEV (Minimal Information for Studies of Extracellular Vesicles) guidelines ${ }^{36}$.

\section{Culture growth}

Cyanobacterial cultures can be readily obtained from one of the many culture collections available worldwide. A few examples are the Roscoff Culture Collection (Station Biologique de Roscoff, France), the Pasteur Culture Collection (Institute Pasteur, Paris, France), and the Provasoli-Guillard National Center for Marine Algae and Microbiota (NCMA, Maine, USA). The cyanobacterial strain of choice must be cultivated in the appropriate medium and environmental conditions, varying significantly among different strains. A list of commonly used media for cyanobacterial cultivation can be found at culture collection websites or other publications $37,38,39$.

Working with cyanobacterial extracellular vesicles presents some unique challenges compared with the methodologies reported for many typical model laboratory heterotrophs. Cyanobacterial cultures have vesicle concentrations of orders of magnitude lower than those found in other microbes such as Escherichia coli ${ }^{14,16,40}$. These differences, presumably arising from lower cell densities and/or vesicle production rates, mean that relatively large cultures $(\sim 1-20 \mathrm{~L}$ or more) may be required to yield sufficient material for bulk analyses. Thus, researchers are encouraged to test vesicle yields from smaller-scale cultures to determine how much material will be necessary to achieve their desired end goals. The importance of establishing whether the media used for the experiment has a detectable particulate background also needs to be stressed before beginning any experiment, as that material can potentially confound vesicle population quantification, reduce the sensitivity of vesicle concentration/size measurements, or contaminate the final vesicle preparations.

Another challenge for the field is that not all cyanobacteria grow well, or at all, in pure culture. Until rendered axenic, interpretations of physical vesicle characteristics, production rates, or contents cannot necessarily lead to clear conclusions about vesicles produced by any one strain in the community. Researchers are also advised to consider what other types of particles could be present and potentially confused with vesicles by specific nanoparticle analysis tools, which cannot necessarily discriminate between different particle types. For instance, it may be essential to verify that the strain being used lacks a prophage, either via genome sequencing, induction assays, or other means or does not release other types of particulate matter. In our experience, most cyanobacterial vesicles are $<0.2 \mu \mathrm{m}$ in diameter, but when looking at a new strain or growth condition, one should confirm whether using a $0.45 \mu \mathrm{m}$ pore-size filter alters the size distribution of purified particles.

Many aspects of culture conditions can influence vesicle production and its contents ${ }^{41,42}$. Thus, the physical and chemical conditions used for culture growth (including light irradiance, temperature, and media composition) need to 
be documented and controlled to the degree possible to ensure the reproducibility of results. Any chemical analysis of vesicle contents must account for background composition, particularly when carrying out '-omics' style high-throughput analyses. This can be especially critical when using undefined media, such as those based on a natural seawater background or supplemented with yeast extract or tryptone. Using a defined growth medium may be preferable depending on the experimental goals.

Researchers need to carefully monitor culture growth dynamics at regular intervals to ensure that they know where in the growth phase a given batch culture is and not just collect samples after an arbitrary amount of time. Vesicle composition can vary across growth phases, particularly between exponential and stationary phases 41,42 . For instance, at least some fraction of vesicles sampled in the stationary phase may arise from a different cellular mechanism, such as cell lysis, that will not occur during exponential growth. While this may still be of great biological interest, it is essential to know the sample. If a cyanobacterial culture reaches the desired growth phase at a time when it is not possible to proceed directly to sample concentration, separating the cells from the $<0.2 \mu \mathrm{m}$ fraction immediately (with centrifugation and/or direct $0.2 \mu \mathrm{m}$ filtration), and then storing the cell-free filtrate at $4{ }^{\circ} \mathrm{C}$ is recommended. The material can be stored in this way for days with little to no noticeable impact on the concentration or size distribution of vesicles.

\section{Vesicle purifications}

The frequent need to isolate vesicles from significant volume cultures is vital in the cyanobacterial vesicle isolation workflow. When working with larger volumes of material, the vesicles will need to be concentrated before downstream separation workflows. Concentrators (tangential flow filter membranes or centrifugal columns) with a nominal molecular weight limit of $100 \mathrm{kDa}$ are generally advised, as they allow separation from soluble material with low molecular weight while keeping concentration times reasonable, but $30 \mathrm{kDa}$ filters are also frequently used with success. While several non-ultracentrifugation-based methods of purifying vesicles (e.g., size exclusion chromatography, microfluidicbased systems, affinity-capture techniques, and precipitationbased approaches) are becoming popular in the extracellular vesicle field, in our experience, these approaches can result in decreased yields and are typically incompatible with the culture volumes needed.

Researchers need to consider the composition of the iodixanol background and the wash/resuspension buffers used during vesicle purification to ensure that they are compatible with the desired downstream applications. In many cases, the final vesicle sample can be resuspended in growth media or a defined buffer comparable in composition to the growth medium (e.g., natural vs. artificial seawater). However, this may not be possible with marine cyanobacterial vesicles, which will require further experimental manipulation for analysis, as high salt concentrations similar to seawater levels can inhibit many enzymatic reactions. In such cases, standard laboratory buffers such as $0.2 \mu \mathrm{m}$ filtered, 1x PBS typically work well for maintaining the stability of marine cyanobacterial vesicles and can be more compatible with downstream experimental processes.

Density gradient purification can be considered optional depending on the experimental goals and culture composition but is strongly recommended for producing a more rigorously pure and reproducible sample. EV populations are heterogeneous and can be found across a range of buoyant 
densities, which further vary by strain, growth conditions, and other factors $4,5,6$. The densities listed above represent those typically found for vesicles from cyanobacterial cultures and field samples in iodixanol, but results in other strains may vary. Other density gradient materials such as sucrose and $\mathrm{CsCl}$ can be utilized for vesicles, but they will migrate to different buoyant densities in these backgrounds. Different gradient media backgrounds can bias the recovery of lipid-enclosed viruses ${ }^{43}$ and might potentially influence the recovery of vesicles from different strains.

Vesicles can be lost at multiple points through the vesicle isolation, and gradient purification process described here, reducing yields and increasing the amount of starting material needed to achieve a given final vesicle yield for downstream applications. Particular care should be taken when working with vesicle pellets following ultracentrifugation. While some cyanobacterial vesicles can have carotenoids or other compounds which may lend vesicle samples some amount of pigmentation (Figure 2), depending on the strain or amount of material, it is not necessarily expected to be able to visualize the vesicles pellet directly. Be aware of where the pellet is expected to be given the type of centrifuge rotor used. When possible, purified vesicle samples are suggested to be examined by electron microscopy to verify the composition of the final material recovered.

The impact of storage conditions on vesicles and their contents remains an open question. Though it is found that the storage does not have a notable effect on cyanobacterial vesicle size or concentration ${ }^{14}$, the functionality of isolated vesicle preparations may change over time ${ }^{44}$. While freeze/ thaw cycles should be avoided whenever possible, the impact of freezing samples on overall vesicle numbers and sizes appears to be minimal. One should be aware of the potential for freeze-thaw cycles to influence the composition of vesicle contents, such as the length of vesicle-associated nucleic acids or the stability of proteins.

\section{Vesicles from field samples}

Current methods for isolating extracellular vesicles from natural aquatic environments are conceptually and operationally similar to those described here for large-volume cultures. Still, they can require even greater volumes of material. Such field samples can involve collecting, filtration, and concentration of hundreds to thousands of liters of water to obtain sufficient material for analysis. Depending on the turbidity of the sample being used, incorporation of one or more pre-filtration steps before the $0.2 \mu \mathrm{m}$ filter may be needed. The specific TFF device used should be appropriate for working with such large volumes in a reasonable amount of time (ideally in the order of hours) and without putting excessive pressure on the sample. In practice, this often involves using a much greater total surface area than might be applied to culture-based samples, as well as larger-diameter tubing to facilitate increased flow rates. This increased filter surface area will likely lead to a marginal increase in particle losses compared to smaller TFF arrangements and a larger final volume of concentrate; however, these concerns must be balanced with considerations of total processing time. For situations such as an extended oceanographic cruise where samples will not return to a lab for many days post-sampling, we recommend carrying out initial $0.2 \mu \mathrm{m}$ filtration and TFF steps in the field. This smaller volume of concentrated material can then be stored at $4{ }^{\circ} \mathrm{C}$ or $-80{ }^{\circ} \mathrm{C}$ on board (depending on availability and downstream analytical considerations) until it is returned to the lab for final processing. 
Isolation and separation of extracellular vesicles from other small particles, both organic and inorganic, can be challenging, and methods for separating different particles are not yet perfect. For instance, iodixanol density gradients may not readily separate all classes of vesicles and viruses present in a given sample. As the types of confounding particles, and their physical properties, will vary between sampling sites, it is currently impossible to provide a protocol that will robustly partition all classes of small aquatic particles. Trial and error are essential, and experimentation with the iodixanol range used in the gradient and ultracentrifugation conditions will be required to maximize the separation; a collection of smaller volume, more finely resolved density fractions may also be required. Depending on the context, using $\mathrm{CsCl}$ gradients instead of iodixanol may help separate environmental particles ${ }^{45}$. Still, the change in osmotic conditions could lead to biases in the final recovered products, as discussed above.

\section{Vesicle characterization}

Nanoparticle analysis instruments are not yet routinely available in microbiology laboratory settings but are becoming increasingly available. All methodologies have pros and cons, and we make no specific endorsement of one platform as being better than all others for cyanobacterial vesicle work; indeed, all have particular trade-offs concerning costs, resolution, ease of use, detection limits, compatibility with different growth media/ buffer backgrounds, and data reproducibility. In addition to instruments based on nanoparticle tracking analysis described above, other approaches, including nanoflow cytometry, microfluidic resistive pulse sensing, and tunable resistive pulse sensing' can be applied ${ }^{46,47}$. Users should be careful to learn the details of their available instrumentation and verify that it works well with their system, as we have encountered difficulties when using some platforms with seawater-based media. We encourage the field to move toward quantitative characterization of vesicle size, concentrations, and production rates. Measuring vesicle concentrations on a fundamental particle per $\mathrm{mL}$ basis, and not in terms of protein content or other metrics, will allow for the integration of vesicles into more quantitative frameworks and enable intercomparisons among strains and conditions. Further efforts to improve the ability to calibrate concentration measurements for $<100 \mathrm{~nm}$ particles are needed.

The fact that vesicle production rate measurements are done directly from $0.2 \mu \mathrm{m}$ filtered culture supernatant to minimize particle losses would be associated with other purification steps described above. However, this does mean that the approach does not necessarily discriminate between actual extracellular vesicles and other small particles found in the cultures. Only counting particles within specific size ranges (e.g., 50-250 nm diameter) may help exclude some outliers, but visual confirmation that pelleted $<0.2 \mu$ m culture contents appear to be membrane-bound vesicles (by TEM or other approaches) is needed to be able to specifically claim that one is measuring the production of vesicles as opposed to the production of vesicle-like particles.

An essential factor in vesicle characterization is to ensure that the vesicle sample is being analyzed in the appropriate linear sensitivity range of the nanoparticle analysis device. When a sample is too concentrated, it is straightforward to dilute that material with a clean buffer and re-analyze it. On the other hand, the relatively low cell and/or vesicle density of some cyanobacterial cultures can sometimes yield vesicle preparations that are below some instruments' detection limits. In cases where this occurs with bulk vesicle purifications, one can consider growing larger volume 
cultures, re-pelleting and resuspending the final material in a smaller volume, or evaluating whether there is a step in the isolation process where excessive losses are occurring and could be mitigated. When measuring vesicle production rates, the fixes are not necessarily so straightforward. Samples can be concentrated if necessary, but the first one should see whether adjustments to the media could result in higher cell densities or whether sufficiently concentrated samples could be obtained from later time points during the exponential phase where concentrations will be higher.

\section{Limitations}

As with any protocol, vesicle isolation using these approaches has clear limitations. These approaches do not rely on their guarantee that an utterly pure preparation of vesicles will be isolated. Both cultures and field samples may contain other materials, which migrate similarly to vesicles in density gradients. Still, at a minimum, these types of additional purification methodologies are essential for ensuring rigor and reproducibility of vesicle analysis. While we describe vesicle isolation approaches in the context of cyanobacteria, cultures of many other microbes will also contain vesicles at comparatively low concentrations, and the procedures described here should be generally applicable. It is expected that these methods will serve not as a permanent protocol but instead as a starting point for spurring future advancements in working with extracellular vesicles from diverse microbes. Future efforts are required to merge these methods with other approaches such as size exclusion columns or asymmetric field flow fractionation to improve the discrimination and separation of different categories of small particles from cultures and environmental samples. We are also hopeful that these techniques can continue to evolve alongside improvements in nanoparticle characterization technologies to improve the ability to examine heterogeneity within vesicle populations, their contents, and their exact functional roles in the environment.

\section{Disclosures}

The authors declare no conflicts of interest.

\section{Acknowledgments}

The authors acknowledge the support of the i3s Scientific Platforms "Biointerfaces and Nanotechnology", and "Histology and Electron Microscopy", a member of the national infrastructure Portuguese Platform of Bioimaging (PPBI-POCI-01-0145-FEDER-022122). We also thank Prof. J. A. Gonzalez-Reyes (University of Córdoba, Spain) for helping optimize the ultra-thin section staining protocol for TEM, and Dr. Cecília Durães and Dr. Ana Rita Pinto (University of Porto, Portugal) for the nanoparticle tracking analysis.

This work was funded by the US National Science Foundation (OCE-2049004 to SJB), by Fundo Europeu de Desenvolvimento Regional (FEDER) funds through the COMPETE 2020 Operacional Programme for Competitiveness and Internationalisation (POCI), Portugal, 2020, and by Portuguese funds through Fundação para a Ciência e a Tecnologia/Ministério da Ciência, Tecnologia e Ensino Superior in the framework of the project POCl-01-0145-FEDER-029540 (PTDC/BIAOUT/29540/2017 to PO). Fundação para a Ciência e a Tecnologia is also greatly acknowledged for the PhD fellowship SFRH/BD/130478/2017 (SL), and FCT Investigator grant IF/00256/2015 (PO). M.C.M.-M. was supported by a Marie Skłodowska-Curie Individual Fellowship (Reintegration panel) within the Horizon 2020 Framework Programme (H2020-MSCA-IF-2018-RI-844891). 


\section{References}

1. Raposo, G., Stoorvogel, W. Extracellular vesicles: Exosomes, microvesicles, and friends. Journal of Cell Biology. 200 (4), 373-383 (2013).

2. van Niel, G., D'Angelo, G., Raposo, G. Shedding light on the cell biology of extracellular vesicles. Nature Reviews Molecular Cell Biology. 19 (4), 213-228 (2018).

3. Coelho, C., Casadevall, A. Answers to naysayers regarding microbial extracellular vesicles. Biochemical Society Transactions. 47 (4), 1005-1012 (2019).

4. Schwechheimer, C., Kuehn, M. J. Outer-membrane vesicles from Gram-negative bacteria: biogenesis and functions. Nature Reviews Microbiology. 13 (10), 605-619 (2015).

5. Toyofuku, M., Nomura, N., Eberl, L. Types and origins of bacterial membrane vesicles. Nature Reviews Microbiology. 17 (1), 13-24 (2019).

6. Kulp, A., Kuehn, M. J. Biological Functions and Biogenesis of Secreted Bacterial Outer Membrane Vesicles. Annual Review of Microbiology. 64 (1), 163-184 (2010).

7. Berman-Frank, I., Lundgren, P., Falkowski, P. Nitrogen fixation and photosynthetic oxygen evolution in cyanobacteria. Research in Microbiology. 154 (3), 157-164 (2003).

8. Flores, E., Herrero, A. Compartmentalized function through cell differentiation in filamentous cyanobacteria. Nature Reviews Microbiology. 8 (1), 39-50 (2010).

9. Flombaum, P. et al. Present and future global distributions of the marine cyanobacteria Prochlorococcus and Synechococcus. Proceedings of the National Academy of Sciences of the United States of America. 110 (24), 9824 (2013).

10. Biller, S. J., Berube, P. M., Lindell, D., Chisholm, S. W. Prochlorococcus: the structure and function of collective diversity. Nature Reviews Microbiology. 13 (1), 13-27 (2015).

11. Abed, R. M. M., Dobretsov, S., Sudesh, K. Applications of cyanobacteria in biotechnology. Journal of Applied Microbiology. 106 (1), 1-12 (2009).

12. Lea-Smith, D. J. et al. Editorial: Exploring the growing role of cyanobacteria in industrial biotechnology and sustainability. Frontiers in Microbiology. 12, 1963 (2021).

13. Garcia-Pichel, F., Zehr, J. P., Bhattacharya, D., Pakrasi, H. B. What's in a name? The case of cyanobacteria. Journal of Phycology. 56 (1), 1-5 (2020).

14. Biller, S. J. et al. Bacterial vesicles in marine ecosystems. Science. 343 (6167), 183 (2014).

15. Pardo, Y. A., Florez, C., Baker, K. M., Schertzer, J. W., Mahler, G. J. Detection of outer membrane vesicles in Synechocystis PCC 6803. FEMS Microbiology Letters. 362 (20) (2015).

16. Oliveira, P. et al. The versatile TolC-like SIr1270 in the cyanobacterium Synechocystis sp. PCC 6803. Environmental Microbiology. 18 (2), 486-502 (2016).

17. Biller, S. J. et al. Membrane vesicles in sea water: heterogeneous DNA content and implications for viral abundance estimates. The ISME Journal. 11 (2), 394-404 (2017).

18. Yin, H. et al. Synechococcus elongatus PCC7942 secretes extracellular vesicles to accelerate cutaneous wound healing by promoting angiogenesis. Theranostics. 9 (9), 2678-2693 (2019). 
19. Lima, S., Matinha-Cardoso, J., Tamagnini, P., Oliveira, P. Extracellular vesicles: An overlooked secretion system in cyanobacteria. Life. 10 (8), 129 (2020).

20. Gupta, S., Marcela Rodriguez, G. Isolation and characterization of extracellular vesicles produced by iron-limited mycobacteria. Journal of Visualized Experiments: JoVE. (152), e60359 (2019).

21. Jung, A. L. et al. Legionella pneumophila outer membrane vesicles: Isolation and analysis of their pro-inflammatory potential on macrophages. Journal of Visualized Experiments: JoVE. (120), e55146 (2017).

22. Fantappiè, L. et al. Antibody-mediated immunity induced by engineered Escherichia coli OMVs carrying heterologous antigens in their lumen. Journal of Extracellular Vesicles. 3 (1) (2014).

23. Moore, L. R. et al. Culturing the marine cyanobacterium Prochlorococcus. Limnology and Oceanography: Methods. 5 (10), 353-362 (2007).

24. Rippka, R. et al. Prochlorococcus marinus Chisholm et al. 1992 subsp. pastoris subsp. nov. strain PCC 9511, the first axenic chlorophyll a2/b2-containing cyanobacterium (Oxyphotobacteria). International Journal of Systematic and Evolutionary Microbiology. 50 (5), 1833-1847 (2000).

25. Moore, L. R., Chisholm, S. W. Photophysiology of the marine cyanobacterium Prochlorococcus: Ecotypic differences among cultured isolates. Limnology and Oceanography. 44 (3), 628-638 (1999).

26. Partensky, F., Blanchot, J., Vaulot, D. in Marine Cyanobacteria. Eds Charpy, L., Larkum, A.W.D. 457475 Bulletin de l'Institut Océanographique de Monaco. (n spécial 19) (1999).
27. Stanier, R. Y., Kunisawa, R., Mandel, M., Cohen-Bazire, G. Purification and properties of unicellular blue-green algae (order Chroococcales). Bacteriological Reviews. 35 (2), 171-205 (1971).

28. Fomsgaard, A., Freudenberg, M. A., Galanos, C. Modification of the silver staining technique to detect lipopolysaccharide in polyacrylamide gels. Journal of Clinical Microbiology. 28 (12), 2627-2631 (1990).

29. Abramoff, M.D., Magalñaes, P.J., and Ram, S.J. “Image processing with ImageJ", Biophotonics International. 11 (7):36-42, (2004).

30. Peterson, T. Densitometric analysis using NIH image. North American Vascular Biology Organization (NAVBO) eNewsletter. 16 (3) (2010).

31. Jürgens, U. J., Weckesser, J. Carotenoid-containing outer membrane of Synechocystis sp. strain PCC6714. Journal of Bacteriology. 164 (1), 384-389 (1985).

32. Whitfield, C., Trent, M. S. Biosynthesis and export of bacterial lipopolysaccharides. Annual Review of Biochemistry. 83 (1), 99-128 (2014).

33. McBroom, A. J., Johnson, A. P., Vemulapalli, S., Kuehn, M.J. Outer membrane vesicle production by Escherichia coli is independent of membrane instability. Journal of Bacteriology. 188 (15), 5385-5392 (2006).

34. Kadurugamuwa, J. L., Beveridge, T. J. Virulence factors are released from Pseudomonas aeruginosa in association with membrane vesicles during normal growth and exposure to gentamicin: a novel mechanism of enzyme secretion. Journal of Bacteriology. 177 (14), 3998-4008 (1995).

35. Nguyen, T. T., Saxena, A., Beveridge, T. J. Effect of surface lipopolysaccharide on the nature of 
membrane vesicles liberated from the Gram-negative bacterium Pseudomonas aeruginosa. Journal of Electron Microscopy. 52 (5), 465-469 (2003).

36. Théry, C. et al. Minimal information for studies of extracellular vesicles 2018 (MISEV2018): a position statement of the International Society for Extracellular Vesicles and update of the MISEV2014 guidelines. Journal of Extracellular Vesicles. 7 (1), 1535750 (2018).

37. Norena-Caro, D. A., Malone, T. M., Benton, M. G. Nitrogen sources and iron availability affect pigment biosynthesis and nutrient consumption in Anabaena sp. UTEX 2576. Microorganisms. 9 (2), 431 (2021).

38. Rippka, R. Isolation and purification of cyanobacteria. Methods in Enzymology. Academic Press. 167, 3-27 (1988).

39. Van Alphen, P., Abedini Najafabadi, H., Branco dos Santos, F., Hellingwerf, K. J. Increasing the photoautotrophic growth rate of Synechocystis sp. PCC 6803 by identifying the limitations of its cultivation. Biotechnology Journal. 13 (8), 1700764 (2018).

40. Reimer, S. L. et al. Comparative analysis of outer membrane vesicle isolation methods with an Escherichia coli tolA. mutant reveals a hypervesiculating phenotype with outer-inner membrane vesicle content. Frontiers in Microbiology. 12 (383) (2021).

41. Zavan, L., Bitto, N. J., Johnston, E. L., Greening, D. W., Kaparakis-Liaskos, M. Helicobacter pylori growth stage determines the size, protein composition, and preferential cargo packaging of outer membrane vesicles. Proteomics. 19 (1-2), 1800209 (2019).

42. Soares, N. C. et al. Associating growth-phase-related changes in the proteome of Acinetobacter baumannii with increased resistance to oxidative stress. Journal of Proteome Research. 9 (4), 1951-1964 (2010).

43. Thurber, R. V., Haynes, M., Breitbart, M., Wegley, L., Rohwer, F. Laboratory procedures to generate viral metagenomes. Nature Protocols. 4 (4), 470-483 (2009).

44. Dell'Annunziata, F. et al. Outer membrane vesicles derived from Klebsiella pneumoniae are a driving force for horizontal gene transfer. International Journal of Molecular Sciences. 22 (16), 8732 (2021).

45. Linney, M. D., Schvarcz, C. R., Steward, G. F., DeLong, E. F., Karl, D. M. A method for characterizing dissolved DNA and its application to the North Pacific Subtropical Gyre. Limnology and Oceanography: Methods. 19 (3), 210-221 (2021).

46. Arab, T. et al. Characterization of extracellular vesicles and synthetic nanoparticles with four orthogonal singleparticle analysis platforms. Journal of Extracellular Vesicles. 10 (6), e12079 (2021).

47. Cimorelli, M., Nieuwland, R., Varga, Z., van der Pol, E. Standardized procedure to measure the size distribution of extracellular vesicles together with other particles in biofluids with microfluidic resistive pulse sensing. PLOS ONE. 16 (4), e0249603 (2021). 